\title{
CoviD-19 Respiratory outpatient clinics during and post COVID-19: the use of patient satisfaction and preference data to redesign service delivery
}

\author{
Authors: Malvika Bhatnagar, ${ }^{\mathrm{A}}$ Paul McCallion, ${ }^{\mathrm{B}}$ John Davison, ${ }^{\mathrm{C}}$ Tony DeSoyza ${ }^{\mathrm{D}}$ and Katy Hester $^{\mathrm{E}}$
}

\begin{abstract}
We used a survey to evaluate patient satisfaction with a remote system for the delivery of respiratory clinics and to capture future preferences. $98 \%$ of responders were satisfied with their current appointment being held remotely in order to reduce the risks from COVID-19. Regarding future preferences beyond the pandemic, $41 \%$ of respondents preferred a faceto-face appointment, $35 \%$ preferred a remote appointment and $24 \%$ had no preference of one modality over another. Additional qualitative data suggest that a flexible system allowing patients to choose the mode of attendance shortly before the appointment would be welcomed.
\end{abstract}

KEYWORDS: respiratory clinics, remote consultation, patient satisfaction and preference, redesign service

DOI: 10.7861/fhj.2020-0181

Background and methods

The COVID-19 pandemic has altered medical service delivery, but also provided an opportunity to examine our practices and determine areas requiring improvement. The Royal College of Physicians (RCP) and Royal College of General Practitioners (RCGP) released guidance on resetting outpatient services in the context of the pandemic using quality improvement methods. I In keeping with guidance, we conducted a study to evaluate a remote clinic delivery system by capturing patient satisfaction with the new system and their future preferences, with the aim of informing redesign of service delivery post-COVID-19.

We converted multiple respiratory clinics to telephone or video consultations. At the conclusion of remote appointments, patients

Authors: AST3 respiratory medicine, Newcastle upon Tyne Hospitals

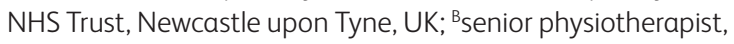
respiratory medicine, Newcastle upon Tyne Hospitals NHS Trust, Newcastle upon Tyne, UK; ' senior nurse specialist for complex lung diseases, Newcastle upon Tyne Hospitals NHS Trust, Newcastle upon Tyne, UK; Dhonorary consultant physician, Newcastle upon Tyne Hospitals NHS Trust, Newcastle upon Tyne, UK; É consultant respiratory physician, Newcastle upon Tyne Hospitals NHS Trust, Newcastle upon Tyne, UK

\begin{tabular}{lll}
\hline \multicolumn{2}{ll}{ Table 1. Characteristics of patients surveyed } \\
\hline Characteristic & Options/measures & Results [N=269] \\
Clinic 'attended' & Consultant general & 22 \\
& Consultant & 76 \\
& bronchiectasis & \\
& Consultant COPD & 57 \\
& Specialist nurse clinic & 73 \\
& Physiotherapist clinic & 41 \\
New referral & New & $83(31 \%)$ \\
or review & Review & $186(69 \%)$ \\
Distance from & Miles (median/range) & $5(<1-166)$ \\
hospital & & \\
Age & Years (median/range) & $69(19-94)$ \\
Diagnosis/ & Bronchiectasis & $239(67 \%)$ \\
reason for & COPD & $39(11 \%)$ \\
referral & Bronchiectasis/asthma & $24(6 \%)$ \\
& Bronchiectasis/COPD & $18(5 \%)$ \\
& Cough & $17(5 \%)$ \\
& Asthma & $11(3 \%)$ \\
& SOB & $9(2 \%)$ \\
Call duration & Other & $2(1 \%)$ \\
\hline CoPD & Minutes (median/range) & $14(1-42)$ \\
\hline
\end{tabular}

$\mathrm{COPD}=$ chronic obstructive pulmonary disease; $\mathrm{SOB}=$ shortness of breath.

( $n=269$, see Table 1 ) were asked a series of questions regarding their consultation and future preferences. Data were collected from multiple clinics, including four consultant-led clinics (a general respiratory clinic, two bronchiectasis clinics and a COPD clinic), two specialist nurse clinics (severe asthma and bronchiectasis clinics), and one physiotherapist clinic with mainly bronchiectasis patients.

Results

We achieved a response rate of $92 \%$ (248/269), with $98 \%$ of responders being satisfied with their current appointment being held remotely. When asked about future preferences for appointments, $41 \%$ of respondents preferred a face-to-face appointment when safe 
to do so, $35 \%$ preferred a remote appointment (29\% telephone, $6 \%$ video), and $24 \%$ had no preference of one modality over another.

\section{Discussion}

Physical footfall in clinics could, therefore, potentially be reduced by almost $60 \%$ without adversely affecting patient satisfaction. Exploration of patients' preferences for future clinic formats revealed an overarching theme of 'wanting choice of clinic format', wherein patients could choose the mode of attendance, with 'perceived wellness' being a common theme influencing their decisions. These qualitative data suggest that incorporating systems that facilitate conversion between modalities as the appointment nears would seem to be the optimum approach. This would further improve patient satisfaction and most appropriate allocation and uptake of clinic slots.

Our response rate was high, perhaps due to concurrent data collection at conclusion of the remote appointment. This is, therefore, likely to describe patient preferences more accurately than studies with lower response rates. The data also reflect a mixed patient population from multidisciplinary clinics.

This project focused on patient preferences. More work to ascertain clinical effectiveness and identification of suitable patients and schedules for facilitating remote services safely is needed.

\section{Conclusions}

The conversion of respiratory outpatient appointments to remote consultations was well received within our cohort. Being able to provide a significant proportion of multidisciplinary patient appointments remotely allows for potential reductions in costs, travel time and environmental impacts. It also has potential to reduce waiting time for those requiring face-to-face assessment, for example, those with severe asthma or being investigated for lung cancer who may be more suited to an in-person appointment. Reducing overcrowding in waiting rooms decreases risks of infection even beyond COVID-19. Most patients wanted choice of mode of attendance closer to the appointment. Redesigning services with these features will be a big step towards improving efficiency of outpatient services in line with patient preferences and novel ways of working post-COVID-19.

\section{Reference}

1 Royal College of Physicians. Rebuilding the NHS - Resetting outpatient services for the 21st century in the context of COVID-19. RCP, 2020. Available from www.rcplondon.ac.uk/guidelines-policy/ rebuilding-nhs-resetting-outpatient-services-21st-centurycontext-covid-19.

Address for correspondence: Dr Katy LM Hester, Cardiothoracic Directorate, Freeman Hospital, Newcastle upon Tyne Hospitals NHS Trust; Freeman Road, High Heaton, Newcastle upon Tyne, NE7 7DN UK.

Email: katy.hester@nhs.net 